\title{
Numerical Analysis of Effective Permeability of Concrete-Like Material With a Random Three-Phase Mesostructure
}

\begin{abstract}
Shibing Zhang ${ }^{1}$, Zhen Sun ${ }^{2}$, Junli Zhou ${ }^{3}$, Bo Yang ${ }^{4}$, Jiuchang Zhang ${ }^{5,6 *}$ and Zhihao Cui ${ }^{7}$
${ }^{1}$ Binhai Hydraulic Engineering Construction Corporation Limited, Yancheng, China, ${ }^{2}$ No. 6 Gas Production Plant, Changqing Oilfield Company, PetroChina, Yan'an, China, ${ }^{3}$ Shenhua Shendong Quality Technical Inspection and Test Center, Ordos, China, ${ }^{4}$ Oil Production Plant 6 of Petrochina Changqing Oilfield Company, Xi'an, China, ${ }^{5}$ Yunnan Provincial Key Laboratory of Civil Engineering Disaster Prevention, Kunming University of Science and Technology, Kunming, China, ${ }^{6}$ Department of Civil Engineering, Yunnan Minzu University, Kunming, China, ${ }^{7}$ Research Institute of Geotechnical Engineering, Hohai University, Naniing, China
\end{abstract}

Interfacial transition zone (ITZ) is an important component of a concrete-like material. Accurately simulating the ITZ's characteristics of the concrete-like materials is a difficult process in numerical simulation. This article proposed a random three-phase mesostructural modeling method using the incorporation of random aggregate generation, Minkowski sum theory, and polygon union techniques. It was found that this method can better simulate the mesostructure and ITZ characteristics of concrete-like materials. By using this method, a random three-phase mesostructural model had been built for conducting a finite element analysis to investigate the effective permeability parameters of concrete. A good agreement between numerical and experimental results indicates the feasibility of this method in the concrete-like material analysis.

Keywords: effective permeability, Minkowski sum, interfacial transition zone, random three-phase mesostructure, concrete-like material

\section{INTRODUCTION}

Concrete is regarded as a composite material composed of aggregates, cement mortar, and an interfacial transition zone (ITZ) between it. The concept that an ITZ exists around sand and coarse aggregate particles in concrete has been one of the accepted tenets of concrete technology for many years [1]. Due to low strength, low elastic modulus, and high permeability, the ITZ is considered to be the weakest area of concrete. To some extent, the ITZ is the main factor which determines the mechanical properties of concrete.

How to accurately describe the characteristics of the ITZ is significant in the study of concrete mechanical behavior. Compared with the simple analytical investigation, numerical simulation technology can be considered to be competent for the mechanical description of complex morphological ITZ characteristics of concrete-like materials [2]. By establishing a random aggregate mesoscale model which can reflect the aggregate gradation, content, and morphology, the performance index of each phase of concrete can be obtained with a numerical simulation. The application of numerical simulation has gradually deepened the understanding of the mesomechanical behavior of concrete-like materials [3]. 


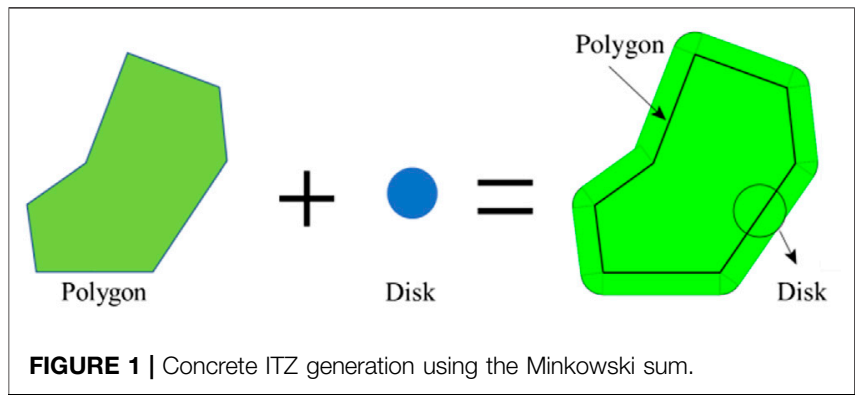

At present, there are four main ways to model an ITZ of concrete. The first method is to treat the concrete aggregates as a polygon and to extend around the polygon $[4,5]$. However, when the particles have sharp corners, this method overestimates the range of the ITZ. The second method is using the same cubic elements and setting the boundary element of aggregates as that of the ITZ [6]. This solution is easy to use but the geometry of the ITZ cannot be described accurately. The third approach is to treat the ITZ as a thicknessfree interface element and set a weak parameter for the ITZ [7-9]. However, two problems exist in this method. The parameters of the thickness-free interface element are difficult to determine, and the time step will be very small due to large stiffness of the cohesive element. The fourth method is realized by the Minkowski sum theory, which can reasonably determine the ITZ [10-12]. This method can describe the geometry of the ITZ accurately and can set different thicknesses easily. However, most of the particles used in the current research are convex, and there are only a few studies that use concave particles.

This research proposed a three-phase random mesostructure model generation method consisting of an aggregate, a matrix, and an ITZ. A Minkowski sum algorithm for the concave aggregate is employed to calculate the ITZ. Based on the random mesostructural model established using this method, a finite analysis had been conducted to study the effect of the ITZ on the effective permeability coefficient of concrete.

\section{METHODS}

\section{Aggregate Generation}

There are many methods to generate aggregates, like point extension [13], Fourier transform [14], and spherical harmonics [15]. In this article, a simple random perturbation, which was proposed by the previous study, is employed [16,17]. The procedure of the rock aggregate generation is described as follows:

1) Generate a set of random length sequences $\left\{r_{1}, r_{2}, r_{3}, \ldots, r_{n}\right\}$ :

$$
\begin{gathered}
r_{i}=r_{0}+(2 \varsigma-1) \Delta r \\
\varsigma=\operatorname{rand}(0,1), \Delta r=\operatorname{rand}\left(0, r_{0}\right) .
\end{gathered}
$$

2) Generate a set of random angle increments $\left\{\Delta \theta_{1}, \Delta \theta_{2}, \Delta \theta_{3}, \ldots, \Delta \theta_{n}\right\}$, where the range of $\Delta \theta_{i}$ is from $-2 \pi /$ $n$ to $2 \pi / n$ :

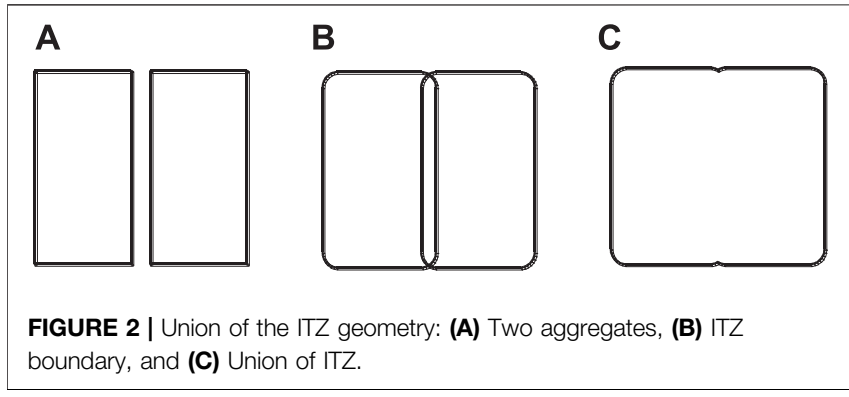

$$
\Delta \theta_{i}=\frac{2 \pi}{n}+(2 \varsigma-1) \eta \frac{2 \pi}{n}
$$

where $\eta$ is a coefficient between 0 and 1 .

3) Memorize all $\Delta \theta_{i}$ which are equal to $360^{\circ}$, the $\Delta \theta_{i}$ are processed following Eq. 3 and then updated to the sequence as follows:

$$
\Delta \bar{\theta}_{i}=\Delta \theta_{i} \frac{2 \pi}{\sum \Delta \theta_{i}} .
$$

4) Convert the polar coordinate system into the Cartesian coordinate system; the following coordinates are obtained:

$$
x_{i}=r_{i} \cos \left(\sum_{j=1}^{i} \Delta \bar{\theta}_{j}\right) y_{i}=r_{i} \sin \left(\sum_{j=1}^{i} \Delta \bar{\theta}_{j}\right),
$$

In the above five equations, the subscript " $i$ " means the corresponding variables at the $i$-th point for generating a polygon.

\section{Minkowski Sum-Based ITZ}

Minkowski sum is named after the great German mathematician Hermann Minkowski. It is used as the sum of two convex point sets. The Minkowski sum of point set A and B is defined as follows:

$$
A \oplus B=\{a+b \mid a \in A, b \in B\} .
$$

It is noted that the original Minkowski sum is only suitable for the convex polygon. For two concave polygons, a set of convex

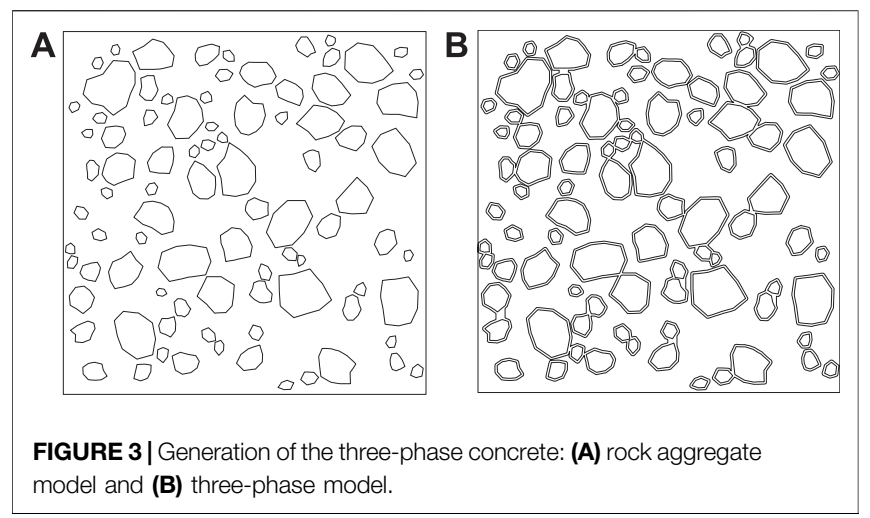



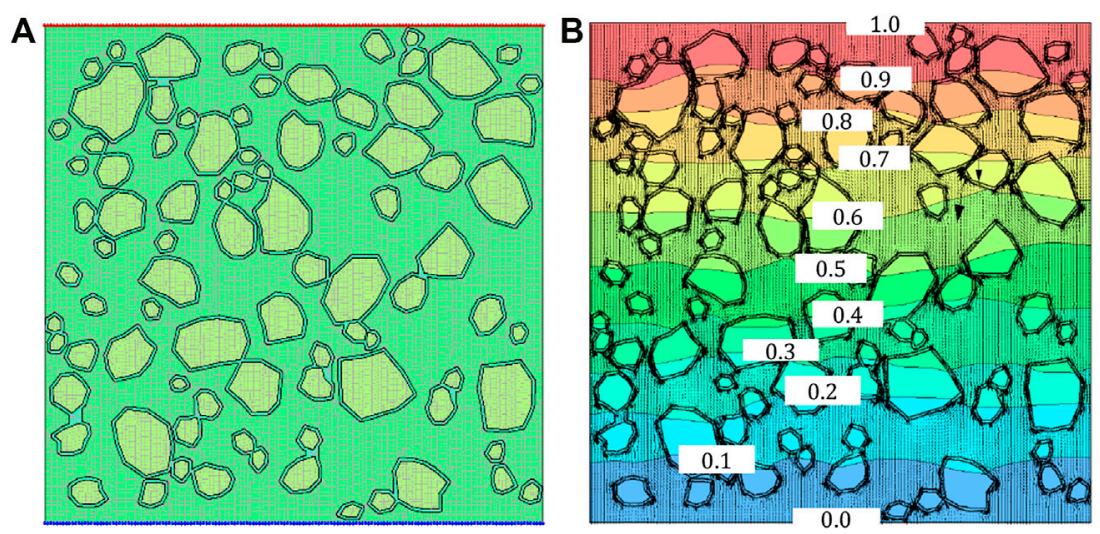

FIGURE 4 | FEM simulation of the three-phase concrete: (A) mesh model and (B) FEM simulation result.

sub-polygons is obtained using the convex decomposition method.

We then calculate the pairwise sums using a simple procedure for the Minkowski sum and finally compute the union to get the final shape. As for the generation of the ITZ of concrete, we can add the concave aggregate with a small disk as shown in Figure 1.

\section{Three-Phase Mesostructure Model Random Aggregate Placement}

Random aggregate placement includes particle generation, trial placement, judging intersections, adjusting positions, and determining the placement. The detailed process is given as follows:

1) Randomly select a particle size in the range of the particle gradation sizes. If the aggregates tend to arrange, rotate the particles to the specified inclination angle.

2) Transfer the block randomly into a box and judge whether it intersects with the placed block; polygon contact analysis is a well-studied problem in computational geometry.

3) If they do not intersect, the placement of this block is determined. If the result of the block intersection is obtained in step (2), re-determine the placement position of the block and repeat step (2) until the placement is confirmed.

\section{ITZ Union}

After the ITZ of concrete is generated, a new problem arises. The rock aggregate is non-overlapping as shown in Figure 2A. After the Minkowski sum of a disk is calculated, the ITZ geometry will be connected (Figure 2B). In this study, we simply use a union algorithm in Matlab to put together the connected areas into one area, as shown in Figure 2C.

\section{Concrete Model Generation}

The random generation of concrete is based on a procedure which is commonly called the "take-and-place method" [18]. Based on the analysis mentioned above, there are mainly five steps proposed for the model generation, stated as follows:
1) The size segment and size distribution of aggregates are determined based on the proposed method.

2) Generate a random aggregate initially and reshape it according to properties such as elongation, direction, and smoothness.

3) Place the aggregates in the domain while considering the border effect. If there is no overlap with the previously generated rock blocks, new rock aggregates will be generated one-by-one in this way.

4) Implement the Minkowski sum with a small disk with a radius of the ITZ 's length for all the rock aggregates.

5) Merge the ITZ geometry and write the information of the ITZ and rock aggregates in the geometry software. In this study, the "dxf" file format was adopted in AutoCAD.

\section{RESULTS}

To validate the feasibility of this method, we refer to a laboratory test by [19] to analyze the effective permeability of concrete. A cubic concrete sample ( $\mathrm{Ls}=100 \mathrm{~mm}$ ) having 88 aggregates in a volume fraction of $40 \%$ is randomly generated, as shown in Figure 3A. After adding a disk with a radius of $0.5 \mathrm{~mm}$ using the Minkowski sum and union operation, the three-phase concrete model is built as shown in Figure 3B.

In this study, GeoStudio is employed to calculate the permeability of the three-phase concrete. Considering this model to be a multiply-connected region, the three-phase model cannot be imported directly into GeoStudio. We first collect the centroids of all particles and plot the ray line. In this way, the whole model can be cut into a lot of slices. In this way, the multiply-connected region is divided into a simply connected region that can be imported into GeoStudio in the format of a closed polyline. Afterward, we re-assign the group for each area that can generate the final model.

The permeability parameter is referred from previous studies. The coefficient of the permeability of mortar is readily obtained from the laboratory experiment as $k_{m}=1.95 \times 10^{-2} \mathrm{~mm} / \mathrm{s}$, and the aggregates are assumed to be impermeable (e.g., 
$k_{a}=1.95 \times 10^{-9} \mathrm{~mm} / \mathrm{s}$ ). The permeability coefficient of the ITZ is defined as $k_{i t z}=\lambda k_{m}$, and the coefficient is set as 10 .

After the permeability parameter for each material is assigned, a seed density of $0.5 \mathrm{~mm}$ is used to mesh the model. To compute the effective coefficient of concrete, a numerical constant water head with a height of $1 \mathrm{~m}$ is employed as shown in Figure 4A.

Based on the numerical simulation results of the seepage field (Figure 4B), the effective permeability of concrete is estimated with a value of $6.73 \times 10^{-3} \mathrm{~mm} / \mathrm{s}$. The value of the laboratory test result is $6.21 \times 10^{-3} \mathrm{~mm} / \mathrm{s}$. The error between the numerical and laboratory test values is $8.37 \%$. The result indicates that the proposed numerical model can give a good estimation of the permeability of concrete.

\section{CONCLUSION}

This work proposed a three-phase model generation method for concrete-like materials using the Minkowski sum theory. This method can give an accurate description of the ITZ geometry. The numerical simulation result of FEM shows a good agreement with the experimental parameters. This study indicated that the ITZ is a very important factor which affects the property of the concretelike material.

Besides, this method provides a numerical tool for the analysis of percolation. With the variation in the ITZ's thickness and parameters, a more interesting result will be obtained. As a

\section{REFERENCES}

1. Diamond S, and Huang J. The ITZ in concrete - a Different View Based on Image Analysis and SEM Observations. Cement and Concrete Composites (2001) 23:179-88. doi:10.1016/S0958-9465(00)00065-2

2. Meng Q-X, Xu W-Y, Wang H-L, Zhuang X-Y, Xie W-C, and Rabczuk T. DigiSim - an Open Source Software Package for Heterogeneous Material Modeling Based on Digital Image Processing. Adv Eng Softw (2020) 148: 102836. doi:10.1016/j.advengsoft.2020.102836

3. Bentur A, and Alexander MG. A Review of the Work of the RILEM TC 159ETC: Engineering of the Interfacial Transition Zone in Cementitious Composites. Mat Struct (2000) 33:82-7. doi:10.1007/bf02484160

4. Wang J, Fan Y, Fan Y, Zhu C, Lu S, and Liu J. Mesoscopic Finite Element Simulation on the Interfacial Bonding Performance of Functionally Gradient Concrete. ES Mater Manuf (2020). doi:10.30919/esmm5f810

5. Yang W. Modeling Recycled Aggregate concrete Crack by Extended Finite Element Method and concrete Damage Plasiticity (2016).

6. Yu Q, Liu H, Yang T, and Liu H. 3D Numerical Study on Fracture Process of concrete with Different ITZ Properties Using X-ray Computerized Tomography. Int J Sol Structures (2018) 147:204-22. doi:10.1016/ j.ijsolstr.2018.05.026

7. Maleki M, Rasoolan I, Khajehdezfuly A, and Jivkov AP. On the Effect of ITZ Thickness in Meso-Scale Models of concrete. Construction Building Mater (2020) 258:119639. doi:10.1016/j.conbuildmat.2020.119639

8. Wang X, Yang Z, and Jivkov AP. Monte Carlo Simulations of Mesoscale Fracture of concrete with Random Aggregates and Pores: a Size Effect Study. Construction Building Mater (2015) 80:262-72. doi:10.1016/ j.conbuildmat.2015.02.002

9. Wang X, Zhang M, and Jivkov AP. Computational Technology for Analysis of 3D Meso-Structure Effects on Damage and Failure of concrete. Int J Sol Structures (2016) 80:310-33. doi:10.1016/j.ijsolstr.2015.11.018

10. Xu W, Jia M, Zhu Z, Liu M, Lei D, and Gou X. n-Phase Micromechanical Framework for the Conductivity and Elastic Modulus of Particulate Composites: Design to Microencapsulated Phase Change Materials common technique, this study has a broad application prospect in the future.

\section{DATA AVAILABILITY STATEMENT}

The original contributions presented in the study are included in the article/Supplementary Material, further inquiries can be directed to the corresponding author.

\section{AUTHOR CONTRIBUTIONS}

The conception and design of the study was mainly contributed by SZ and JZ. The programming code was mainly contributed by SZ and ZS. The analysis and/or interpretation of data was mainly contributed by BY and JZ. Numerical simulations were mainly contributed by JZ and ZC. Drafting the manuscript was mainly contributed by SZ, ZS.

\section{FUNDING}

This study was financially supported by the Fundamental Research Funds for the Central Universities (B200201059) and China Postdoctoral Science Foundation (Grant No. 2020M673589XB).

(MPCMs)-Cementitious Composites. Mater Des (2018) 145:108-15. doi:10.1016/j.matdes.2018.02.065

11. $\mathrm{Xu} \mathrm{W,} \mathrm{Wu} \mathrm{Y,} \mathrm{and} \mathrm{Jia} \mathrm{M.} \mathrm{Elastic} \mathrm{Dependence} \mathrm{of} \mathrm{Particle-Reinforced}$ Composites on Anisotropic Particle Geometries and Reinforced/weak Interphase Microstructures at Nano- and Micro-scales. Compos Structures (2018) 203:124-31. doi:10.1016/j.compstruct.2018.07.009

12. Zhu Z, Provis JL, and Chen H. Quantification of the Influences of Aggregate Shape and Sampling Method on the Overestimation of ITZ Thickness in Cementitious Materials. Powder Tech (2018) 326:168-80. doi:10.1016/ j.powtec.2017.12.008

13. Xu W-j., Zhang H-y., Jie Y-x., and Yu Y-z.. Generation of 3D Random MesoStructure of Soil-Rock Mixture and its Meso-Structural Mechanics Based on Numerical Tests. J Cent South Univ (2015) 22:619-30. Journal of Central South University. doi:10.1007/s11771-015-2563-1

14. Mollon G, and Zhao J. Fourier-Voronoi-based Generation of Realistic Samples for Discrete Modelling of Granular Materials. Granular Matter (2012) 14: 621-38. doi:10.1007/s10035-012-0356-x

15. Wei D, Wang J, Nie J, and Zhou B. Generation of Realistic Sand Particles with Fractal Nature Using an Improved Spherical Harmonic Analysis. Comput Geotechnics (2018) 104:1-12. doi:10.1016/j.compgeo.2018.08.002

16. Meng QX, Wang HL, Xu WY, and Cai M. A Numerical Homogenization Study of the Elastic Property of a Soil-Rock Mixture Using Random Mesostructure Generation. Comput Geotechnics (2018) 98:48-57. doi:10.1016/ j.compgeo.2018.01.015

17. Meng Q, Wang H, Cai M, Xu W, Zhuang X, and Rabczuk T. Threedimensional Mesoscale Computational Modeling of Soil-Rock Mixtures with Concave Particles. Eng Geology (2020) 277:105802. doi:10.1016/ j.enggeo.2020.105802

18. Wang ZM, Kwan AKH, and Chan HC. Mesoscopic Study of concrete I: Generation of Random Aggregate Structure and Finite Element Mesh. Comput Structures (1999) 70:533-44. doi:10.1016/s0045-7949(98) 00177-1

19. Li X, Xu Q, and Chen S. An Experimental and Numerical Study on Water Permeability of concrete. Construction Building Mater (2016) 105:503-10. doi:10.1016/j.conbuildmat.2015.12.184 
Conflict of Interest: SZ was employed by Binhai Hydraulic Engineering Construction Corporation Limited; ZS was employed by Changqing Oilfield Company, PetroChina; and BY was employed by the Oil Production Plant 6 of Petrochina Changqing Oilfield Company.

The remaining authors declare that the research was conducted in the absence of any commercial or financial relationships that could be construed as a potential conflict of interest.

Publisher's Note: All claims expressed in this article are solely those of the authors and do not necessarily represent those of their affiliated organizations or those of the publisher, the editors, and the reviewers. Any product that may be evaluated in this article, or claim that may be made by its manufacturer, is not guaranteed or endorsed by the publisher.

Copyright (c) 2021 Zhang, Sun, Zhou, Yang, Zhang and Cui. This is an open-access article distributed under the terms of the Creative Commons Attribution License (CC $B Y)$. The use, distribution or reproduction in other forums is permitted, provided the original author(s) and the copyright owner(s) are credited and that the original publication in this journal is cited, in accordance with accepted academic practice. No use, distribution or reproduction is permitted which does not comply with these terms 